\title{
LAS ESCLAVAS EN LA REGULACIÓN JURÍDICA. ALGUNAS NOTAS DESDE EL DIGESTO'.
}

\author{
Female slaves in the legal regulation -notes from Digest
}

\author{
Carla Rubiera Cancelas \\ Universidad de Oviedo. \\ E-mail: carlarubiera@gmail.com \\ Fecha de recepción: 6-III-2011 \\ Fecha de aceptación: 22-III-2011
}

\begin{abstract}
Resumen: No cabe duda de que estudio de la legislación nos brinda la oportunidad de analizar el funcionamiento de la esclavitud en la sociedad romana. Sin embargo, nos encontramos con que los propios juristas partirán de la premisa: "La palabra esclavo se extiende también a la esclava" (D. 50, 16, 40 Ulp. ed), lo que llega a suponer un problema en el momento en el que nos interesemos por la población esclava femenina, en el sentido de que el uso de un lenguaje, que hoy podría catalogarse como sexista, no nos permite diferenciar entre unos y otras. Sin embargo, en determinadas ocasiones observaremos la utilización de términos como el de ancilla o serva. Al margen de la aparente homogeneidad que caracterizó al grupo esclavo, mi interés se centra en indagar en esa presencia femenina dentro de una fuente jurídica, que en este caso constituye la gran recopilación del derecho romano, el Digesto, buscando las particularidades que pudieron afectar a las esclavas, no tanto como pertenecientes a una clase, sino a un género, el femenino.
\end{abstract}

Palabras clave: esclavitud femenina, género, Digesto, sociedad romana antigua

ABSTRACT: There can be no doubt that the study of legislation offers the opportunity to analyse the functioning of slavery in Roman society. However, even jurists point out that the word slave not only includes male but also female slaves. The use of this term -which nowadays can be regarded as sexist- can be problematic when focusing the female slave population since it is not possible to differentiate between both sexes. Nevertheless, terms such as ancilla or serva are sometimes used to establish a difference. Regardless of the apparent homogeneity which characterises the slave class, my research focuses on finding female slave population in one of the biggest legal resources in Roman law, the Digest. My aim is to study the particularities that might have affected female slaves due not only to their belonging to a particular class but also to a particular gender.

Keywords: female slavery, gender, Digest, roman society

1 Este artículo se enmarca dentro del proyecto I+D, "Claves diacrónicas de la divergencia social entre las construcciones simbólicas y las construcciones jurídicas de la maternidad". Ref. HAR 2009-100035-HIST. 


\section{INTRODUCCIÓN}

En el seno de la sociedad romana, inequívocamente, la esclavitud tiene una gran importancia y presencia. No en vano, uno de los juristas más relevantes del imperio dirá en sus Instituciones, "La principal división en el derecho de las personas es esta; que todos los hombres son libres y esclavos" (D. 1, 5, 3 Gayo). Así, la población esclava aparecerá muy abundantemente en las fuentes del mundo antiguo, especialmente en las jurídicas, lo que nos permitirá observar la regulación del funcionamiento del sistema esclavista, pudiendo además percibir los problemas que surgen en lo cotidiano, en los encuentros y desencuentros entre el grupo libre y esclavo.

Parece, por tanto, relativamente fácil obtener información sobre la esclavitud, sin embargo, si intentamos discernir las diferencias existentes dentro de la clase esclava, atendiendo al género, surgen una serie de problemas derivados en su mayoría de la caracterización de las fuentes que vayamos a utilizar. En este sentido, lo habitual es hacer referencia a la invisibilización femenina a través del masculino neutro y del androcentrismo. Tales aspectos constituyen una dificultad importante para aquellas personas que intenten indagar en el pasado femenino de manera general y más particularmente en el de las esclavas.

Precisamente, por todo ello, considero significativo el momento en el que en las fuentes jurídicas encontramos el término esclava, utilizando por tanto el género femenino en una clase de textos que nos recuerdan: "La palabra esclavo se extiende también a la esclava" [D. 50,16,40,1, (Ulp. 56 ed.) ]. Indagar en el porqué de esa presencia femenina y en su justificación constituye el principal objetivo del presente escrito, penetrando así en la aparente homogeneidad que encierra la esclavitud.

2. EL DERECHO COMO FUENTE PARA EL ESTUDIO DE LA ESCLAVITUD FEMENina. Algunos problemas

No constituye ninguna novedad que las fuentes jurídicas son de gran importancia para el estudio de la sociedad romana antigua debido a que nos brindan la oportunidad única de analizar la configuración del ordenamiento social, económico, religioso, político y la legitimación del mismo. Además, nos permiten observar el funcionamiento de un sistema de género en esta misma sociedad, como bien manifiesta la obra de Jane Gardner, Women in the roman Law and Society (1991). La desigualdad legal que se percibe a través de la lectura de la legislación, queda perfectamente resumida en la afirmación de Papiano, cuando dice que "en muchos extremos de nuestro derecho es peor la condición de las hembras que la de los varones" [D. 1,5,9 (31, quaest)], lo que responde a un ordenamiento social no igualitario, que por otra parte viene justificado.

Justamente, por esta razón, he seleccionado el Digesto, obra elaborada por mandato de Justiniano en el siglo VI d. C., como es sabido, y que 
constituye la gran recopilación del derecho romano, lo que nos puede dar una amplia perspectiva del tema que vamos a tratar. Prácticamente todo el texto está impregnado de la estela de la esclavitud, en tanto que ésta constituía una parte fundamental del mundo romano de la antigüedad. Se manifiesta así este escrito absolutamente interesante para el estudio de la esclavitud de manera general, permitiéndonos acceder e interpretar los problemas derivados del funcionamiento de un sistema esclavista.

Si atendiésemos a lo dicho por algunos de los juristas del mundo antiguo podríamos encontrarnos con afirmaciones como la de Celso, quien definió el derecho "como el arte de lo bueno y de lo equitativo". Ulpiano dirá que "la jurisprudencia es el conocimiento de lo divino y de lo humano, la ciencia de lo justo y de lo injusto". Los juristas eran conscientes de las contradicciones de la existencia humana, de las tensiones y su deber era resolverlo. Esto es justamente lo que se refleja en el Digesto. En la gran mayoría de los casos encontraremos instrucciones sobre asuntos bastante concretos y minuciosos, lo cual puede llamarnos la atención, sin embargo, se trataba de resolver lo individual sin perder de vista lo general. Si hablásemos de esclavitud en la sociedad romana de manera amplia, enumerando grandes cuestiones, podríamos decir, el esclavo no tiene capacidad jurídica, no es sujeto de derecho o no tiene capacidad de obrar, entre otras cosas. Ahora bien, si abandonamos esa amplia categoría, y entramos en lo particular nos encontraremos en ocasiones -limitadas- a las ancillae ${ }^{2}$. Precisamente el Digesto nos permite observar como determinados juristas en sus alegatos tuvieron que hablar de estas feminae porque, a pesar de que "lo dicho para el esclavo es también para la esclava", igual que "lo dicho para el varón ha de incluir también a la hembra", la sociedad romana delimita muy claramente los comportamientos de cada género, por lo que tendremos que diferenciar dentro de cada grupo social entre hombres y mujeres ${ }^{3}$.

Hablar de un sistema de género dentro de la esclavitud pudiera resultar contradictorio, pues tanto esclavos como esclavas son catalogados como res corporeae. Sin embargo, la lectura de las fuentes nos permite llegar a la conclusión de que independientemente de la conceptualización del

2En este artículo utilizaré ancillae como sinónimo del serva. No obstante, soy consciente de afirmaciones como la de Marguerita Garrido-Hory, la cual afirma que este término puede hacer referencia a varios estatus. Véase la obra de esta autora: Derecho privado romano. Madrid, Edersa, pp. 35.

3 Sobre la importancia de superar la categoría clase mediante la aplicación del género véase por ejemplo el capítulo de CID LÓPEZ, Rosa Ma: "Joan Scott y la historia de las mujeres en España. El caso de los estudios sobre la antigüedad”. En BORDERÍAS, Cristina (ed.): Joan Scotty las políticas de la bistoria. Barcelona, Icaria, 2006, pp. 61-94. Para el caso de la población esclava, destacando la importancia del género en cuanto a que implica un doble subordinación si estamos hablando de mujeres reducidas a un estatus servil, véase MARTÍN CASARES, Aurelia: "Imaginario y realidad de la esclavitud femenina". En BALLARÍN DOMINGO, Pilar y MARTÍNEZ LÓPEZ, Cándida: Del patio a la plaza. Las mujeres en las sociedades mediterráneas. Granada: Universidad de Granada. Servicio de publicaciones, pp. 155-163. 
esclavo o de la esclava como perteneciente a un grupo social, existen ciertos aspectos que sólo podrían ser explicados si atendemos a la configuración de los roles de género.

Por último, ha de mencionarse que el ordenamiento jurídico está íntimamente ligado con el modelo social en el que nace; el derecho ha sido considerado signo, causa y consecuencia de la organización de la sociedad, tal y como reflexionó Pierre Vilar. No podemos quedarnos en un análisis superficial de las fuentes legislativas, haciendo una lectura que sólo nos permita relacionar nuestro sistema jurídico con el pasado, o que simplemente nos conceda la oportunidad de observar la evolución de determinadas instituciones, estructuras o incluso términos. El derecho refleja la imagen de una sociedad en la que surge y, para ello, debemos de dotar a la información que extraemos de un enfoque histórico, de un contexto que traduzca una serie de artículos en el entendimiento profundo, hasta donde sea posible, -soy consciente de que las fuentes legislativas tienen una limitaciones, las cuales habrán de ser salvadas con la utilización de otras como las epigráficas o literarias-, de una sociedad determinada.

3. El trabajo productivo de las esclavas. Apreciaciones DESDE EL DIGESTO

A medida que se avanza en la lectura del Digesto, se observa la creciente importancia que llegó a tener la esclavitud en la sociedad romana antigua, hasta el punto de impregnar cada uno de los libros que componen la obra que estoy citando. Muy mayoritariamente, cuando se ha investigado sobre la esclavitud se ha tendido a observar su incidencia en la economía de la antigüedad dándonos cifras que, aunque son claramente aproximativas, resultan muy esclarecedoras. Ya en los años ochenta Keith Hopkis, en su obra Conquistadores y esclavos (1981) hablando del modelo de producción esclavista lanza la nada desdeñable cantidad de un treinta por ciento de población esclava durante la etapa imperial. Domenico Vera en un texto más reciente, con el interés de investigar sobre el fin del esclavismo, llega a plantear para la provincia italiana -basándose en un estudio de W. Scheidelel número máximo de un millón quinientos mil esclavos en el siglo I d. C ${ }^{4}$.

Aunque debamos de tener cuidado a la hora de establecer modelos de interpretación globales, la entrada masiva de población esclava a partir de las

4 Quisiera incidir en que este investigador se muestra bastante escéptico a la hora de hacer cálculos sobre la población esclava. Para profundizar más en esta temática véase VERA, Domenico: “Essere schiavi della terra nell' Italia tardoantica: la razionalitá di una dipendenza". En Resistencia, sumisión e interiorización de la dependencia. Studia historica. Historia antigua, vol. 25, 2007, pp. 489-505. Un resumen de las distintas cifras que se han dado para hablar de la población esclava durante época republicana y augustea podemos encontrarlo en ANDREAU, Jean y DESCAT, Raymond: Gli schiavi nel mondo greco e romano. Bologna, Il Mulino, 2009, pp. 62-70. 
guerras de conquista del Mediterráneo (siglo III a. C) acabará propiciando la aparición de un modelo de producción esclavista, que definirá la economía de la sociedad romana y que se mantendrá hasta el siglo II o III d. C dependiendo del autor o autora-, momento en el cual comienza a hablarse de crisis. Por lo tanto, podemos afirmar que el grupo social esclavo representará un porcentaje importante dentro de la población, llegará a monopolizar la mano de obra y su trabajo implicará unos ingresos considerables a las élites, si bien esto quizá no pueda aplicarse a todas las provincias que acabarán configurando el imperio ${ }^{5}$.

Tanto los esclavos como las esclavas constituyen un valor económico en si mismo, el cual se manifiesta a través de su cuerpo y de su fuerza de trabajo y para el caso de las servae además mediante su capacidad de reproducir naturalmente la esclavitud. De nuevo, las fuentes jurídicas pueden arrojar luz a este respecto, por lo que comenzaremos tratando el trabajo productivo para más adelante hablar del reproductivo. El grupo esclavo quedará dividido legalmente en dos categorías familia urbana y familia rustica, las cuales se definirán por el tipo de labor realizada aunque también por el lugar en el que se lleva a cabo ${ }^{6}$. Esta categorización aparece en el Digesto, el cual nos permite además rescatar trabajos realizados por el contingente esclavo, algo que se ha podido comprobar también a través, entre otras fuentes, de la epigrafía.

Ya en la introducción he mencionado una de las dificultades que afectan a las personas investigadoras que quieran acercarse al estudio de las mujeres. La lectura de ciertas partes del Digesto sigue reflejando esos mismos hándicaps. Los "esclavos", "el esclavo" aparecen a lo largo del texto jurídico realizando oficios como el de pintor, tabernero, naviero, establero, trabajador en el campo, pastor, jardinero, y así hasta crear una lista que se alarga infinitamente. Esto constituye un problema porque parece más que probable que, tras esa categoría neutra, en ocasiones podríamos encontrar esclavas, pero la utilización del masculino nos impide afirmarlo. El Digesto regula que lo dicho para el esclavo vale también para la esclava, de igual manera que lo mencionado para el hombre servirá de manera general para la mujer. De todas formas, deberíamos de matizar; debido a que es igualmente cierto que determinados aspectos normalizados para la mulier son aplicables a las servae, si bien hemos de ser conscientes de que no podemos traspasar a la ligera la barrera de la esclavitud. Todo ello se traduce en lo siguiente:

5 Estos parámetros para hablar de una sociedad esclavista son utilizados por BRADLEY, Keith, Esclavitudy sociedad en Roma. Barcelona: Ed. Península, 1998, pp. 25-26.

6 Sobre la familia rustica y urbana puede verse: D. 32, 1, 99 (Ulp. 1 de fideic.), D. 33, 9, 4, 5 (Paul. 4 Sab.) y D. 33, 10, 12 (Lab. 4 pith). 
la esclava pertenece a un grupo social y a un género y ambos aspectos regulan su existencia. Puede dar lugar esta explicación a cierto desconcierto por lo que quisiera citar un ejemplo:

"Pero cuando un esclavo actúa como banquero, cosa posible, supuesto que lo biciese con la voluntad del dueño, ha de ser obligado éste a comunicar la cuenta y ha de darse la acción contra él como si él mismo fuera el banquero." D. 2, 13,4, 3 (Ulp. 4 ed.)

"Las mujeres se considera que quedan excluidas del oficio de banquero, pues es cosa propia de hombres." D. 2, 13,12 (Call. 1 ed. monit.)

Podemos presuponer, en este caso, que la esclava queda excluida de ejercer como banquero, no por una cuestión de clase sino por una cuestión de género ${ }^{7}$.

A pesar de las limitaciones que podamos encontrar a la hora de recuperar el trabajo el trabajo femenino esclavo, es cierto que gracias al Digesto podemos recoger algunos datos, es decir, observaremos tareas en femenino, lo cual tiene una gran importancia partiendo del problema que constituye el masculino neutro. Hasta donde me permiten las lecturas que he hecho, ha de destacarse que las esclavas aparecerán mayoritariamente en el ámbito doméstico, lo que no quiere decir que no pudiesen encontrarse fuera de él; la dificultad radicaría en este caso en que esto, por lo general, no se visibiliza ${ }^{8}$. Quizá de nuevo nos encontramos ante el problema de lo que la sociedad establece o refleja, de acuerdo a un normal entendimiento de lo que son los roles de género frente a lo que ocurre en la realidad, probablemente mucho más cercana a las necesidades y a la obtención de un máximo beneficio? A este respecto hay que señalar que, gracias a estudios recientes que utilizan

7 La figura de la esclava encarna la doble desventaja de pertenecer a un grupo social y a un género fuertemente discriminados. En relación a la pésima consideración que pudiesen tener estas mujeres, analizada desde el punto de vista de las fuentes literarias resulta interesante el texto de GUALERZI, Saverio: "Schiave fedeli sino alla morte". En Resistencia, sumisión e interiorización de la dependencia. Studia historica. Historia antigua, vol. 25, 2007, pp. 203-223

8 Respecto a la falta de reconocimiento de la labora de las mujeres fuera del ámbito doméstico véase MARTÍNEZ LÓPEZ, Cándida: "Las relaciones de género en las unidades domésticas campesinas de la Roma antigua". En MOLAS FONT, M. Dolors (ed.): Vivir en femenino. Estudios de mujeres en la antigüedad. Barcelona, Universitat de Barcelona, 2002, pp. 65-97.

9 En relación a la división de espacios en la obra de Jenofonte resulta interesante el artículo de IRIARTE, Ana: "Fronteras intramuros en el Económico de Jenofonte". En LÓPEZ BARJA DE QUIROGA, Pedro Manuel y REBOREDA MORILLO, Susana (coords.): Fronteras e identidad en el mundo griego antiguo. Santiago de Compostela, Universidad de Vigo. 2001, pp. 267-279. Igualmente merece la pena destacar a este respecto el capítulo de PLÁCIDO, Domingo: "La mujer en el ôkkos y en la pólis: formas de dependencia económica y de esclavización". En REDUZZI MEROLA, Francesca y STORCHI MARINO, Alfredina: Femmes-Esclaves. Modèles d'intreprétation anthropologique, économique, juridique. Atti del XXI coloquio internationale GIREA. Nápoles: Jovene Editrice, 1999, pp. 13-20. 
como fuente principalmente la epigrafía sabemos de mujeres y esclavas que realizaban lo que Francesca Cenerini catalogará como "professioni così topiche, ma anche altre meno connotabili come femminili"; aunque es cierto que no constituyen éstas una gran mayoría, a lo que deberíamos añadir la dificultad de poder evaluar este trabajo ${ }^{10}$.

Realmente, a través de lo leído en el Digesto, sólo nos es posible afirmar, para las esclavas, aquellos oficios que utilicen la fórmula femenina, aunque podríamos intuir otros ${ }^{11}$. En relación a la pregunta de por qué aparecen en femenino determinados labores, podría tener por respuesta que lo realizaban de manera general mujeres y que tradicionalmente no eran concebidos para los varones, aunque quizá pueda deberse a que se está hablando de una ancilla concreta. En el libro treinta y tres, título VII, el cual versa sobre "el legado del fundo dotado con sus pertenencias y el legado de las pertenencias", encontramos a las esclavas como reproductoras pero también como productoras. Desgraciadamente, son pocos los trabajos que aparecen en femenino, pero creo que son suficientes para sacar algunas conclusiones. Tras leer sobre esclavos que cultivan el campo, mayorales y capataces, porteros y barrenderos, barberos y panaderos, encontramos a la esclava que cuece el pan, a las que sirven en la casa, a las que trabajan en la lana y a las que cuidan el fuego ${ }^{12}$. Las esclavas como trabajadoras de la lana aparecerán varias veces en el Digesto, realizando por tanto la labor femenina por excelencia, encargándose como mínimo de las vestimentas que habrían de llevar el resto de los esclavos y esclavas en el fundo, labor que nos recuerdan agrónomos como Catón o Columela en sus obras y que son propias de las mujeres, independientemente de su clase social ${ }^{13}$. Son citadas

Hablando de fuentes clásicas y en relación a la existencia de esclavas trabajando en el exterior encontramos el famoso texto de Columela perteneciente a su tratado de agricultura:

"En los días lluviosos o cuando los fríos o las heladas no dejaren a las mujeres emplearse al raso en los trabajos rústicos, las lanas estarán preparadas y cardadas prestas a ser trabajadas y asi pueda ella dedicarse con más facilidad a esa tarea y no echárselas a otras. Pues nada perjudicará que su ropa, la de los aperadores y la de los otros esclavos que tengan alguna comisión particular se haya becho en casa, y con esto tendrá menos gravamen el padre de familia" (libro duodécimo, III).

10 Cenerini, Francesca. La donna romana: modelli e realtà. Bologna: il Mulino, 2009, página 269.

11 Esta afirmación parece corroborarse a través de la lectura del siguiente fragmento: "Algunos piensan con razón que en el legado de $<<$ esclavos $>>$ se deben también las esclavas, como nombre comin que contiene ambos sexos, pero nadie duda de que no se deben los esclavos varones en un legado de $<<$ esclavas $>>$ ". D. 32, 80 (Mod. 9 diff).

12 En la legislación, será frecuente encontrar a las esclavas que trabajan la lana como un trabajo que ellas realizaban, aunque parece claro que lo hacían por el hecho de ser mujeres no por pertenecer a un grupo social.

13 Son varias las obras que hablarían del trabajo de la lana como específicamente femenino. Me gustaría destacar a este respecto el de MOLAS FONT, Dolors: "Los trabajos de las 
igualmente aquéllas que cuidan el fuego o hacen el pan, labores que de nuevo situamos en el ámbito doméstico junto con las que de manera genérica se nos dice que servían la casa. Igualmente, otras menciones relacionadas con el trabajo productivo de las esclavas serían las encargadas de los adornos y las que aparecen como camareras o acompañantes.

Centrémonos ahora en el grupo de las que sirven en el ámbito doméstico. Es cierto, tal y como señala Bradley, que sabemos de esclavos sin ocupación pero es aun más cierto también que con mayor frecuencia hallamos a las esclavas que sirven en la casa o que trabajan en el ámbito doméstico como mujeres sin labor específica, lo que dificulta un poco más el acercarse al trabajo que realizaron estas servae para poder valorarlo más correctamente $^{14}$. De todas formas, atendiendo al título del texto que leemos, son esclavas que encontramos en una propiedad agraria por lo que, a raíz de otras lecturas, podemos suponer que estas mujeres pudieron estar dedicadas a la transformación de alimentos, a trabajos de mantenimiento o incluso a trabajos agrícolas y ganaderos (pequeñas plantaciones y corrales anejos a la casa). Sin embargo, esto no se visibiliza, tal vez porque el trabajo de estas mujeres llega a entenderse como un todo adherido a la naturaleza femenina que no se considera como tal. Quizá en ello influya lo que Cándida Martínez López llamó los distintos ritmos entre hombres y mujeres resaltando que mientras el varón alterna fuerza y descanso la mujer aparece natural y constantemente atareada pero sin labor concreta ${ }^{15}$. Además, es igualmente cierto que la catalogación de los hombres, sean libres o esclavos, en una sociedad tradicional, suele derivarse por su trabajo en el exterior y, por lo tanto, quizá esto pueda responder a que los esclavos tiendan a aparecer encasillados dentro de una tarea.

mujeres y la economía de las unidades domésticas en la Grecia clásica". En Complutum, 2007, Vol. 18, pp. 271-180. Para un análisis de este tema desde un punto de vista epigráfico y literario véase MEDINA QUINTANA, Silvia: "Las mujeres y la producción textil en la Roma antigua. En Cuestiones de género: de la igualdad y la diferencia. No 4, 2009, pp. 51-64.

14 En relación con el trabajo esclavo véase el capítulo cuatro de la obra de BRADLEY, Keith: Esclavitud y sociedad en Roma. Barcelona: Península, 1998. Esta parte incluye una serie de tablas que nos permiten observar precisamente lo que estoy comentando. Este mismo autor utiliza los datos ofrecidos por Susan Treggiari en su artículo: "Domestic Staff at Rome in the Julio-Claudian Period". Histoire sociale=Social bistory, 6 (1973), pp. 241-255, referente a los esclavos de la casa de Livia. Podríamos hacer la misma lectura observando un extenso número de oficios que aparecerían en masculino, mientras que en femenino encontramos a la pedisequa (ayudante femenina), sarcinatrix (zurcidora), unctrix (masajista), quasillaria (hilandera) o tonstrix (peluquera).

15 Para una lectura en mayor profundidad en torno a esta reflexión véase MARTÍNEZ LÓPEZ, Cándida: "Las relaciones de género en las unidades domésticas campesinas de la Roma antigua". En MOLAS FONT, M. Dolors (ed.): Vivir en femenino. Estudios de mujeres en la antigüedad. Barcelona, Universitat de Barcelona, 2002, pp. 65-97. 
De manera general, a la luz de lo que las fuentes jurídicas reflejan, parece claro que las esclavas participaron de la división sexual del trabajo, no distinguiéndose sus labores excesivamente de las que podían realizar las mujeres libres, aunque hemos de ser conscientes de que estamos hablando de estatus diversos ${ }^{16}$. Así, la esclavitud femenina se manifiesta absolutamente rentable a través de la realización de tareas, al menos hasta donde podemos leer, llevadas a cabo muy mayoritariamente en el ámbito del hogar, encajando sus trabajos en el modelo de economía doméstica del mundo romano.

\section{LAS ESCLAVAS COMO REPRODUCTORAS}

A pesar de que no podríamos disociar trabajo productivo y reproductivo me ha parecido oportuno separarlos en cuanto a la importancia que la reproducción natural de la esclavitud tiene. Parece claro y totalmente asumido que si algo justifica la presencia femenina en cualquier fuente del mundo antiguo es la procreación; esta es una realidad que afectó a mujeres libres y esclavas, debido a que la sociedad romana valoró la fecundidad tanto para unas como para otras.

Si al principio comentaba que el cuerpo de un esclavo constituye un valor en si mismo, el beneficio económico del cuerpo de la esclava radica en un elevado porcentaje en tanto que procreador. Es un hecho que la reproducción natural de la esclavitud era un aspecto fundamental, en cuanto a que constituye una de las maneras a través de la cual se conseguía nueva población esclava, quizá la más importante junto con la obtención a través de las guerras de conquista.

Por todo ello, no debe llamarnos la atención que la mayor parte de las veces que aparece el término esclava o esclavas en la legislación lo hace para relacionarse con su descendencia: "sus hijos"/partus ancillae" el nacimiento de nuevas criaturas aumentaba el patrimonio del dominus o de la domina, se fomentará el contubernium ${ }^{18}$, con la misma finalidad que pudiese tener un matrimonio romano, la reproducción, aunque en este caso con un sentido mucho más economicista. Precisamente, esta idea es afirmada por Ulpiano quien comenta que una esclava embarazada está sana debido a que la principal función femenina es aceptar y preservar la concepción (D. 21, 1, $14,1)$. Se entiende, por tanto, que la contribución de la mujer a la sociedad

16 Sobre la división sexual del trabajo relacionado con la sociedad griega y romana véase MARTÍNEZ LÓPEZ, Cándida y MIRÓN PÉREZ, Ma Dolores: "Mujeres esclavas en la Antigüedad: producción y reproducción en las unidades domésticas". Arenal. Revista de historia de las mujeres. 7/1, 2000.

17 En la edición bilingüe del Digesto aparece la palabra partus ancillae mientras que las ediciones castellanas traducen este término no por parto de la esclava sino por sus hijos.

18 Fórmula permitida para el emparejamiento de esclavos, ya que no tenían capacidad de contraer iustum matrimonium. 
la realiza a través de la concepción, por lo que la salud femenina -libre o esclava- estará relacionada con la fecundidad ${ }^{19}$. La hembra será dadora bien de criaturas libres, las cuales estarán bajo la patria potestas del pater familias o de los vernae, los cuales se mantendrán bajo la domenica potestas. A este respecto puede resultar sumamente interesante cómo el cuerpo femenino de una ingenua podrá igualmente ser catalogado como vientre, -así lo recoge la ley- en el cual se gesta lo que por derecho propio pertenece al marido, cuya inspección en caso de divorcio está legítimamente justificada y amparada por las normas jurídicas ${ }^{20}$.

El cuerpo de la serva será entendido como un medio a través del cual el sistema esclavista se mantiene y reproduce, a la vez que permite a quien lo posee incrementar su poder económico. De ahí la importancia que la fecundidad tiene para quien pretenda adquirir ese cuerpo, entendida como un extra o plus añadido, debido a que hemos visto que las esclavas desempeñaron también trabajos productivos. Por ello, se regulará esa capacidad reproductora concebida como un valor y esto se reflejará en el interés que tienen los juristas por normalizar ciertos aspectos relacionados con el cuerpo de las esclavas en relación a su capacidad procreativa, sobre todo en el momento en que reglamentan la compraventa. Veamos algunos fragmentos a este respecto:

Si resulta estéril aquella esclava de la cual se vende el parto, o mayor de cincuenta años, y el comprador lo bubiese ignorado, el vendedor queda obligado por la acción de compra. D. 19, 1, 21 (Paul. 33 ed.).

Se pregunta si es enferma la esclava que siempre da luz a criaturas muertas, y Sabino dice que si es por defecto de la vulva, es enferma. Si se vendiese una esclava embarazada, admiten todos que está sana, pues la primera y principal función de la mujer es aceptar y preservar la concepción. Asimismo en el puerperio se considera sana a la mujer, siempre que nada extraño acontezca que le provoque una enfermedad corporal. Respecto a la esclava estéril, dice Celio que Trebacio

19 Esta idea la habrían manifestado los discípulos de Hipócrates los cuales concluyeron que la esterilidad era el mal absoluto para la mujer y el parto la mejor prueba de su salud. En relación con Hipócrates y sus conclusiones sobre la salud del cuerpo femenino ver KNIBIEHLER, Yvonne: Historia de las madres y de la maternidad en Occidente. Buenos Aires: Nueva Visión, 2001, página 15. Esta misma idea se refleja en el Digesto a lo largo del libro XXI, cuando regulando la compraventa de la población esclava se catalogue la esterilidad femenina como una enfermedad.

20 Me estoy refiriendo en este caso al D. 25, 4, 1, sobre "la inspección del vientre y de la custodia del parto". Esta idea ya ha sido suficientemente tratada por Eva Cantarella en Calamidad ambigua. Madrid: Clásicas, 1991 y Pasado Próximo. Madrid: Cátedra, 1997. Igualmente, las juristas se han hecho eco de la asimilación de las mujeres a cuerpos reproductores, véase NÚÑEZ PAZ, María Isabel: "Progresivo y limitado reconocimiento de la figura materna en derecho romano. De la cesión del vientre al ejercicio de la tutela". En CID LÓPEZ, Rosa $\mathrm{M}^{\mathrm{a}}$ (coord.): Madres y maternidades. Construcciones culturales en la civilización clásica. Oviedo: KRK, 2009, pp. 255-291. 
distingue si es estéril por naturaleza, y entonces es sana, o si lo es por defecto del cuerpo, y entonces no. D. 21, 1, 14 (Ulp. 1 ed. Aed. Curul.).

La que menstrúa dos veces al mes no es sana, como tampoco la que no menstrúa, salvo que esto se deba a la edad. D. 21, 1, 15 (Paul. 11 Sab).

Consta que no se considera sana la mujer $<$ de pelvis $>$ tan estrecha que no pueda ser madre. D. 21,14,7 (Ulp. 1 ed. Aed. Curu).

La lectura parece clara, pelvis estrechas, falta de menstruación, defecto de vulva, edad, esterilidad; todo ello se traduce en incapacidad o dificultad para la reproducción. Así, el embarazo y el parto son signo de salud corporal, pero también social y económica porque permiten que el sistema esclavista continúe perpetuándose.

He comentado que la lectura del Digesto puede llamar la atención, en cuanto a que da la impresión de que incide en aspectos demasiado particulares. Los juristas con unos conocimientos legislativos aplican o comentan la ley atendiendo aquellos problemas individuales que suponían enfrentamientos o fricciones. En relación con la figura de la esclava resulta altamente significativo, aunque no desligado del tema que tratamos, que aparte de todas las menciones en relación a su capacidad reproductora, se aluda con frecuencia a su descendencia, llegando ésta a alcanzar una mayor importancia que su progenitora. Los vernae tienen un importante valor quizá tanto o más que sus matres, en tanto que constituyen la perpetuación de la esclavitud. Por ello, a lo largo del Digesto, parecen vislumbrarse varios debates en torno a la posesión de esa descendencia en momentos en que pudo existir cierto vacío legal o determinada problemática. Me refiero por una parte a la famosa discusión de si es fruto o no el "hijo" 21 de una esclava, lo que parece ser se zanjó entendiendo que la esclava no es cosa fructífera, por lo tanto el partus ancillae pertenecerá al dueño y no al usufructuario. Otras fueron las preocupaciones en torno a las esclavas y su progenie, como por ejemplo dar respuesta a preguntas tales como si una esclava da a luz incrementa o no la herencia, o si el "hijo" de una serva puede ser adquirido por usucapión o qué pasa con la criatura de una ancilla hurtada.

Podríamos decir que, aunque se esté hablando de las esclavas, realmente lo que se protegen son los intereses económicos de un "poseedor". Parece quedar esto también bastante claro en la primera vez que se habla de las esclavas en el Digesto. Esta inicial mención no se refiere a ellas sino de nuevo a su descendencia. Los juristas se cuestionan sobre lo siguiente: ¿es libre el "hijo" de una esclava habiendo sido concebido en libertad de su madre? o, si habiendo sido la madre esclava da a luz siendo libre ¿es su "hijo" igualmente libre? Otra de las preguntas sería: ¿Si una mujer va a ser manumitida y da a luz, ese "hijo" es libre? Ulpiano responde afirmativamente a los tres interrogantes. De todas formas, a pesar de que se menciona a mujeres reducidas a un estatus de esclavitud, da la sensación de que lo que realmente

21 Utilizo el masculino neutro porque así aparece en la fuente legislativa. 
preocupa a los legisladores no es tanto la mater como la criatura, debido a que sobre ésta pudiese existir, en determinados momentos, alguna duda respecto a su estatus social o su potestad.

\section{A MODO DE CONCLUSIÓN}

La lectura hecha de una parte del Digesto pone de relieve que la aparición de las esclavas en la legislación de la sociedad romana responderá al valor económico y al entendimiento social de su figura, en donde entraría la conceptualización de género. De todas formas, la presencia en femenino de la esclavitud es realmente escasa debido a que los textos jurídicos a través de la utilización del masculino neutro la invisibilizan, algo que quizá nunca podamos recuperar para una comprensión más profunda y exacta de lo que fue la esclavitud.

Los juristas romanos recogerán la experiencia femenina de la población esclava en tanto que pertenecen a un grupo social que constituyó la base de la economía de la antigüedad, resaltando por lo tanto su valor económico, el cual se manifiesta a través de su trabajo productivo y reproductivo. Partiendo de esto, no obstante, podemos atender a la plasmación de un sistema de género configurado en el estrato social más bajo de la sociedad romana.

Respecto al trabajo productivo surgen varias cuestiones. La primera es que se relaciona con el ámbito doméstico, aunque incluso dentro de este espacio es difícil llegar a contabilizarlo debido a que tiende a desaparecer detrás de la fórmula "las que sirven en la casa". La segunda es que la escasa aparición del trabajo de las esclavas tampoco da pie, hasta el momento, a grandes conclusiones, exceptuando que muchos de los trabajos que realizan serían compartidos por las mujeres libres; véase el trabajar la lana. Igualmente, hemos de tener en cuenta que parece más que probable la existencia una serie de actividades que las ancillae no podrían realizar por el hecho de ser feminae. De todas formas, los textos legislativos son una de las fuentes de las que se puede servir la historia, existiendo muchas más que podrían ayudarnos a crear una panorámica más amplia del trabajo femenino esclavo.

En relación a la reproducción de la esclavitud, no cabe duda de que las esclavas jugaron un papel muy importante. Su cuerpo es un objeto, un vientre que se germina para supervivencia del propio sistema. Por ello, la salud de estas servae se ligará a su capacidad procreadora, imagen que no se desliga de la de una mujer libre y así se refleja en los textos que regulan la sociedad. Todo ello, al fin y al cabo demuestra que la experiencia de las mujeres esclavas no fue la misma que la de los varones. Podemos partir de categorías globales a la hora de analizar la esclavitud en la sociedad romana, mencionando la cosificación a la que fueron sometidas millones de personas, analizando los argumentos racionales que se utilizaron para 
crear una otredad respecto a un sujeto dominante que es un varón, libre y ciudadano. Ahora bien, lo leído en el Digesto, hace reflexionar sobre la idea de que dentro de la esclavitud a pesar de que en la lógica romana nos encontramos con res corporeae, al menos creemos oportuno mencionar que a partir de sus tareas y de la conceptualización de sus cuerpos, unas son femeninas y otras masculinas y eso tiene una significación social y, como puede verse en el caso de la esclavitud, también legal.

\section{FUENTES}

Lucio Junio Moderato Columela. Los doce libros de agricultura. (Traducción y notas por Carlos J. Castro: con notas prologadas de Emiliano M. Aguilera). Barcelona: Iberia, 1959.

Digesto (Versión castellana de A. D’Ors, F. Hernández- Tejero, P. Fuenteseca, M. García-Garrido y J. Burillo). Pamplona: Aranzadi, 1975.

Cuerpo del Derecho Civil romano T. I Instituta-Digesto (Traducción y compilación por Ildefonso L. García del Corral). Barcelona: Lex Nova, 2004. 
